\title{
Adaptive Multielement DFE Receivers for Narrowband Multiuser Detection*
}

\author{
Tracy Fulghum \\ Ericsson, Inc. \\ fulghum@rtp.ericsson.se
}

\author{
Alexandra Duel-Hallen \\ N.C. State University
}

\begin{abstract}
The performance of adaptive multielement decision feedback equalization (DFE) receiver structures is examined for a narrowband, two-user space division multiple access (SDMA) system with a TDMA slot structure similar to that of the Digital Advanced Mobile Phone Service (DAMPS). Various means of coupling or cross-feedback of decisions between user receiver sections at the base station are explored, as well as a slot-to-slot adaptive receiver structure. It is found that the cross-feedback of decisions between user receiver sections is an effective means of regaining diversity benefits lost due to the addition of a user to the narrowband system.
\end{abstract}

\section{Introduction}

With the dramatic increase in demand for wireless systems and services in the past few years, there has been a desire to increase the capacity of systems while maintaining or improving the quality of service for the user. Efficient utilization of the bandwidth resources is an important part of capacity issue. CDMA, FDMA, and TDMA are different access methods for exploiting the various dimensions of the signal. Recently, there has been an increased interest in the use of antenna arrays and multielement receivers to exploit the spatial dimension of received signals as well. Multiple co-channel signals can be detected simultaneously within the same time slot with a multielement/antenna array receiver. Detection of multiple, spatially separated signals, with antenna arrays has been alternately termed space division multiple access (SDMA) $[1,2]$ or channel reuse within cell (CRC) [3]. In a flat fading environment, the receiver structure is simply one that weights the received signals at each antenna so that the signal of interest is maximized, while the interfering signal(s) are minimized or "nulled out." This receiver structure is repeated for each user in the SDMA system. However, in the cellular radio environment, the channel often exhibits frequency selective multipath. Intersymbol interference (ISI) must now be contended with, as well as interference from multiple symbols of the co-channel signals (time-dispersed CCI). That is, if there are $J$ interfering signals, and each has two paths in its multipath channel, there are $2 J$ interfer-

*This work was supported in part by the following: Center for Advanced Computing and Communication-NCSU, ORAU, and IBM. The authors would like to thank Karl Molnar of Ericsson Inc. for his helpful comments. ing signals which must be mitigated. This can be accomplished with the addition of antennas and receiver branches (a costly, hardware intensive proposition) or the addition of time processing as well [3].

In [4], Winters et al discuss analytically the use of diversity arrays in an SDMA system. In that paper, both flat fading and frequency selective fading channels are studied and diversity arrays are used for reception. For reception under conditions of a frequency selective channel, linear equalizers are examined for ISI and CCI mitigation. It is shown that the cost of receiving additional users is a degree of diversity per user: for a $J$ user system with $M$ diversity antennas, the diversity benefit for each user is $M-J+1$. For example, with a $M=2$ antenna case receiving two users, $J=2$, each user will receive the diversity benefits of only one branch (i.e., no diversity at all).

This paper examines adaptive multielement decision feedback receiver structures for simultaneously receiving co-channel TDMA transmissions, also presented in [5]. Section 2 of this paper discusses the receivers investigated and the various decision feedback structures. Section 3 presents simulations of these receiver structures and discusses the transmission format and channel models used. Performance results are presented for the receivers in time invariant, slow, and rapidly fading frequency selective channels. We conclude with Section 4.

In our work, we focussed on receivers for diversity arrays. While phased arrays can be used for SDMA receivers, in our case we are more interested in the performance gains that a diversity array can provide rather than the direction finding capability of a phased array receiver. Of particular interest is the use of decisions fed from one user receiver to another, or the coupling of decisions between receivers, to mitigate time dispersed co-channel interference (CCI) and regain diversity lost due to the introduction of additional users. TDMA is an entrenched technology, so it is of particular interest to see if it is possible to alter existing systems to allow for SDMA and increase existing capacity. The receiver structures here are evaluated for a transmission format like that in the Digital Advanced Mobile Phone System (DAMPS).

\section{Receiver Structure}

Spatial-temporal processing basically makes use of equalization structures on each antenna to do the joint task of optimum combining of antenna signals and the mitigation of time dispersed interference: ISI from the time dispersion 


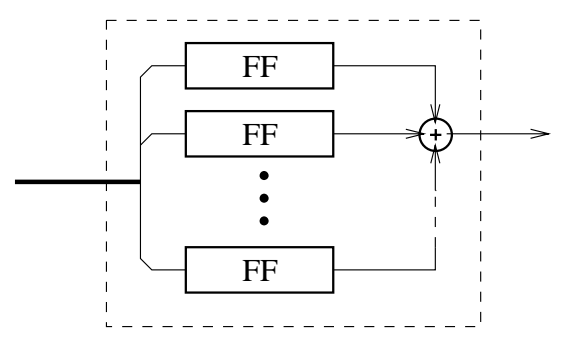

Figure 1: Spatial-Temporal Processor (STP)

of the desired signal, and time dispersed CCI from other users.

The most straightforward use of spatial/temporal processing is shown in Figure 1, where a transversal filter (denoted FF) is placed on the output of each receiver element, and the output of these filters summed. This has been termed the "Spatial-Temporal Processor" (STP) for our purposes.

For resistance to errors in timing phase, and for optimum matched filtering, these delay elements are fractionally spaced. In the case of this work, the elements are spaced at $\frac{T}{2}$, where $T$ is the symbol duration. The weights for these can be determined based on a number of criteria, with combining for minimum mean square error (MMSE) with the desired signal being a common method. However, with multipath of the wireless channels, there exist potential spectral nulls in the channel. As with the linear equalizer, the STP, when used by itself, suffers from a sensitivity to spectral nulls, which makes the introduction of some form of decision feedback into the receiver structure necessary $[6]$.

The basic multielement DFE receiver structure is shown in Figure 2, where two receivers are shown at the same base station. The function of the STP in the receiver is for optimum combining of the antennas for CCI reduction, and for cancellation of the precursor (from "future" symbols) portion of the ISI. The feedback portion of the receiver (the "FB" block) cancels the postcursor portion of the ISI, or that ISI due to past symbols. It is noted that if we have only one receive antenna, this receiver structure reduces to the classic DFE receiver structure, with a fractionally spaced feedforward filter.

The STP has the capability of canceling the time dispersed CCI, but in MMSE adaptation, the CCI is treated as if it were Gaussian interference. No use is made of the structure of the QPSK data transmission inherent in the interference. The structure of the interference can be exploited. Since the interference that is being canceled is due to a transmitted signal that is also being detected at the same base station (the other users in the up link), we can use the decisions of the the users receivers to help mitigate the time dispersed interference.

This "cross-feedback" is shown in Figure 2 by the dashed line and "XFB" blocks. The XFB filters shown are symbol spaced transversal filters which are used to weight decisions from one receiver section for use in interference cancella-

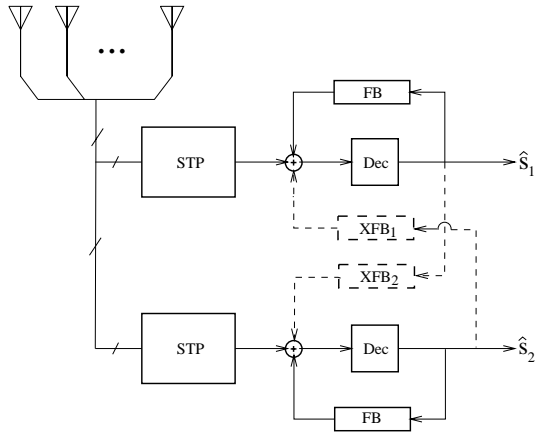

Figure 2: Two User Receiver Structure

tion in the other receiver section.

In the case where only one XFB filter is in place $\left(\mathrm{XFB}_{2}\right.$, where only the decisions from the top section are fed to the bottom section), we have "half-coupling." With this configuration the decisions used for cross-feedback come from the more powerful user for use in the reception of the weaker user, because: (1) There will be more need for the interference canceling capabilities of these decisions in the weaker user's receiver section, and (2) the decisions from the stronger user will be more reliable. For purposes of discussion here, it is assumed that the more powerful user is associated with the top receiver section in Figure 2. If this receiver structure were extended to more than two users, the receiver sections would be ordered so that decisions from other users would only come from more powerful users. This is essentially same concept as in [7], except here in the context of a narrowband, TDMA channel.

In the case where the received signal powers of each user's signal are on the same order of magnitude, there is a relatively significant amount of interference on the stronger user's signal as well. In this case, the use of "full-coupling" is warranted for cancellation of interference in the stronger users receiver section. Here, both XFB filters are in place in 2 .

These receiver structures are similar to the "multivariable DFE" structure as in [8] for multiuser detection for a European cellular (GSM) type transmission format. Here, in the case of half coupling, not every user receiver section utilizes the past decisions of every other user (except in the case of the weakest), and the receiver in this work uses the present decision of other users as well, not just the past decisions ${ }^{1}$. Further, the receiver in that work is not adaptive as in this case, and uses explicit knowledge of the channels for all users to reconstruct the desired signal. Another related work in [9] examines an interference cancellation method using a detected interference signal in a DAMPS-like environment, though not for true multiuser communication.

\footnotetext{
${ }^{1}$ The use of the term "present decisions" presumes that the ordering of receiver processing is such that the present decisions have been made when needed by other receiver sections. This is the case for "future decisions" as well.
} 


\section{Simulation}

For simulation purposes, a DAMPS-like modulation and slot structure was used: a slot of $162 \frac{\pi}{4}$-QPSK symbols transmitted at $25 \mathrm{ksym} / \mathrm{sec}$, with 14 leading symbols used for training purposes. The users had different training sequences of good correlation properties which were chosen from the DAMPS standard [10]. The transmitted symbols were of a root raised cosine (RRC) pulse shape. While not explicitly shown in the figures, RF downconversion is assumed on the signal at each antenna element. The output of the downconversion is receive filtered with an RRC filter, and the rest of the receiver structure is as cited in the previous section.

The weights for each receiver section (STP and decision feedback weights) were solved for adaptively according to an MMSE criterion with the desired signal of that section, using an RLS algorithm with a 0.9 forgetting factor. The adaptation uses the known training sequences as the desired signal during the initial training phase, and thereafter, is decision directed.

Between each user and the base station, there are multiple channels: one between the single transmitter and each receiver element. Each of these channels was modeled as a frequency selective multipath channel with two symbol delayed rays of equal average power. The rays of each channel were independently Rayleigh faded, the fading was independent on each channel as well, suggesting a diversity array configuration for the antennas (widely spaced, $\sim 10$ to $20 \lambda$ separation). The coefficients of these rays are generated using a modified Jakes model [11], and the fading rate of these coefficients is a variable parameter.

We first examine the effect of using the decisions of one user in mitigating the interfering effects on the other user. For this purpose, we use correct decisions in the crossfeedback and the conventional feedback. This allows us to observe effects beyond those due to the feedback of incorrect decisions. In all simulated cases, the performance of the weaker user (associated with the lower section shown in Figure 2) is examined.

The ordering of receiver sections, which section is associated with the stronger user (the top section in Figure 2 ) and weaker user (the bottom section), is based on the the long term average power over many slot transmissions. This is assumed to be known. Further, the "carrier to carrier" ratio or $C / C$, is expressed as the ratio of the long term average received power of the weaker user to the long term average received power of the stronger user.

The STP spanned 2 symbol periods, and the conventional feedback consisted of a single decision fed back within each section. For purposes of observing the effect of cross-feedback, we extended the training period by waiting 85 symbols into the slot before tallying errors, in order to remove effects from adaptation. Also, in this initial observation, we set the fading rate of the channel model taps to be $0 \mathrm{~Hz}$, so that the channel is time invariant and tracking issues are removed.

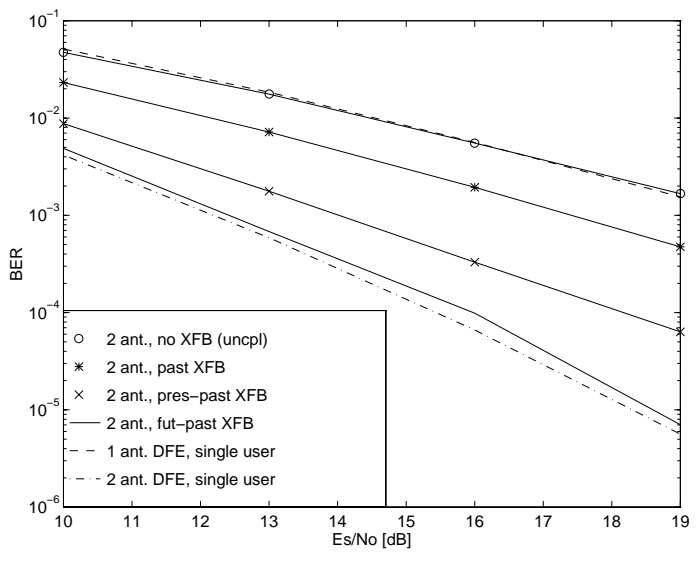

Figure 3: Performance of Coupled and Uncoupled Receivers in Time Invariant, Frequency Selective Environment

Figure 3 shows the performance for a number of two antenna receivers in a time invariant fading channel under equal received power $(0 \mathrm{~dB} C / C)$. In this initial case, since the decisions used in feedback are forced to be correct, only the case of feedback from the stronger user to the "weaker" user receiver section (half-coupling) is considered. For this half-coupling, four cases for the span of the cross-feedback filter over the other user's decision are examined: (1) no decision (no cross-feedback at all, i.e. a conventional DFE diversity array receiver), (2) a single past decision, (3) a single past and the present decision, and (4) a single past, present, and single future decision. For purposes of comparison, the performance of a single antenna DFE and two antenna DFE are shown in Figure 3 under identical conditions except for the fact they are operating with a single user in the system. The uncoupled two antenna receiver performs in the two user system as a single antenna receiver performs in a single user system, verifying that there is a loss of a degree of diversity due to the addition of another user. This represents an extension of the results for linear receivers in [4] to DFE receivers here. Further, as we add more decisions from the other user to span more of the time dispersed interference, the performance gets increasingly better. Ultimately, cross-feedback spanning a single future, present, and single past decision is enough under these particular time dispersion conditions to essentially eliminate the interfering effects of the other user. This two antenna receiver with future-past cross-feedback in a two user system has practically the same performance as a two antenna DFE receiver operating in the same conditions for a single user system, shown also in Figure 3. Essentially, the use of a sufficient span of the other user's decisions in cross-feedback has allowed us to regain that lost degree of diversity.

Next, we examine the effect of half-coupling and fullcoupling, as well as the use of actual decisions on the performance of the weaker long term average power user. Using the same time invariant, frequency selective conditions as before, we simulate the half-coupled and full-coupled re- 


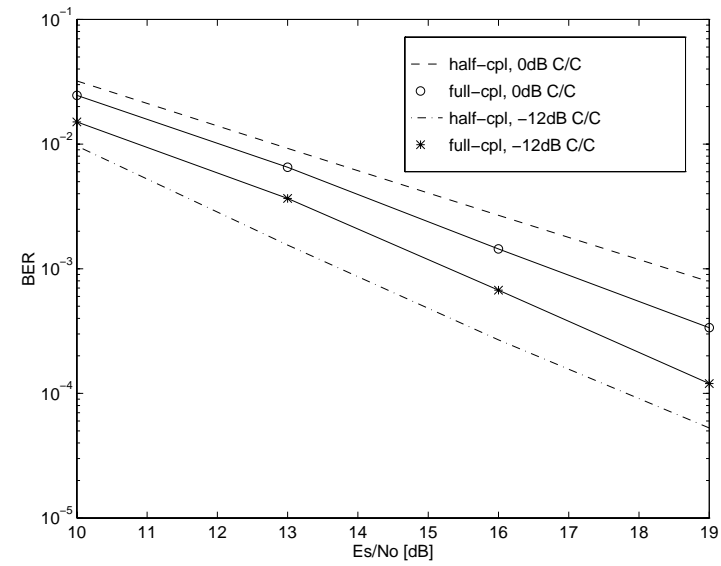

Figure 4: Performance half-coupled and full-coupled DFE receiver sections in $0 \mathrm{~Hz}$ fading

ceiver, with the resulting performance of the weaker user shown in Figure 4. For the half-coupled case, the crossfeedback filter $\mathrm{XFB}_{2}$ only is used, and spans a single future, present, and single past decision. In the full-coupling case, the cross-feedback filter $\mathrm{XFB}_{1}$ is used as well. Due to the order of processing the spans of the filters are restricted. Filter $\mathrm{XFB}_{1}$ is restricted to a span of a single past decision while $\mathrm{XFB}_{2}$ is restricted to a span of the present and a single past decision.

At a $C / C$ of $0 \mathrm{~dB}$, each user experiences the same level of interference on average from the other user. This indicates that both user receivers could make use of the decisions of the other user in mitigating interference. The 0 $\mathrm{dB} C / C$ performance curves of Figure 4 show the effect this full-coupling on the "weaker" user performance. We note a significant impact on the performance with the use of full-coupling. By using the decisions of the weaker user to mitigate interference in the stronger user's receiver processing, more reliable decisions are made there, which are in turn more effectively used to mitigate interference in the weaker user's receiver processing. The $-12 \mathrm{~dB} C / C$ performance curves show that the half-coupled receiver performs better under this condition. Here, while the weaker user is experiencing strong interference, the stronger user is experiencing relatively weak interference. In this case, the decisions from the weaker user in the full-coupled configuration are of less use to the stronger user's receiver, and have less impact on its performance. With little impact on the stronger user's performance, the reliability of its decision is not affected, so this is not reflected in the performance of the weaker user.

Thus, under situations when the powers of the users are close, it is best to use the full-coupled receiver structure, and under situations when the powers are disparate, it is best to use the half-coupled receiver structure. Ultimately, it is desirable to have a receiver structure that adapts itself from slot to slot according to the prevailing conditions of received power. This is implemented in a receiver simulation, where the ordering and coupling of the receiver structure are made according to the received power of each

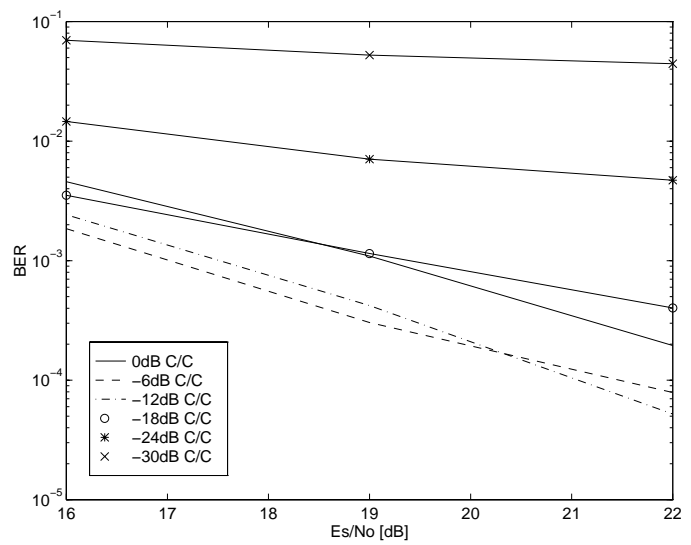

Figure 5: Performance of adaptively structured receiver in $4 \mathrm{~Hz}$ (3 m.p.h), frequency selective environment

user over the course of a slot. If the $C / C$ ratio of weaker to stronger power is below a threshold, then half-coupling structure is chosen with the weaker receiving the decisions of the stronger user. If that ratio is above the threshold, then a full-coupling receiver structure is chosen, with the weaker user receiver section receiving the largest span of decisions from the other user.

Next, this receiver structure is simulated under more realistic fading channel conditions. The frequency selective channel is used as before, but now with a fading rate of $4 \mathrm{~Hz}$, corresponding roughly to a pedestrian speed of 3 m.p.h. at a carrier frequency of $900 \mathrm{MHz}$. The receiver is as before a two antenna receiver with the same STP and conventional feedback structure, except for the adaptive nature of the coupling or cross-feedback. In those cases where half-coupling is chosen, the XFB filter spans a future, present, and past decision from the stronger user. In those case where full-coupling is chosen, strong-to-weak XFB filter spans a present and past decision from the stronger user, and the weak-to-strong XFB filter spans a single past decision from the weaker user. For this slow fading case, the cross-over threshold for switching between receiver structures was determined empirically to be at $C / C=-9 \mathrm{~dB}$. Figure 5 shows the performance over a number of $C / C$ ratios. If we take a minimum performance to be $3 \%$ bit error rate (as in [10]), we see that at a $E_{s} / N_{o}$ of $16 \mathrm{~dB}$, the receiver can operate under $C / C$ ratio conditions of down to $-24 \mathrm{~dB}$.

Finally, the adaptively structured receiver is simulated under rapidly fading rates at $81 \mathrm{~Hz}$, a rate corresponding to a vehicle moving at $60 \mathrm{~m} . \mathrm{p} . \mathrm{h}$. at a carrier of $900 \mathrm{MHz}$. Going to a faster fading situation, the channels change significantly over the course of a slot. This was found to necessitate the use of additional diversity antennas to get acceptable performance. Figure 6 illustrates the performance of the second user (the weaker long term average power) for an adaptively structured receiver with a four element diversity array. The threshold for switching between fullcoupled and half-coupled was determined empirically to be 


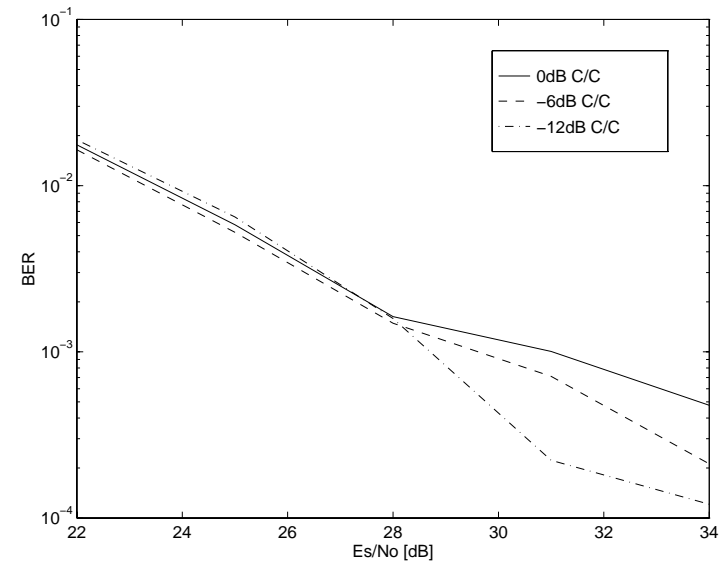

Figure 6: Performance of adaptively structured receiver in $81 \mathrm{~Hz}$ (60 m.p.h), frequency selective environment

$-13 \mathrm{~dB}$. We see that this adaptively structured receiver maintains an acceptable performance for $C / C$ 's down to $-12 \mathrm{~dB}$ and beyond at $E_{s} / N_{o}=22 \mathrm{~dB}$.

\section{Conclusion}

The performance of adaptive multielement decision feedback equalization structures has been examined in a narrowband, two-user space division multiple access (SDMA) system with a TDMA slot structure similar to that of U.S. digital cellular (DAMPS). The results show that the use of one users decisions to mitigate interference in the receiver processing of the other user can significantly improve the performance of the other user's receiver. Winters et al stated that the addition of a user to a SDMA system costs the other users a degree of diversity in their linear receivers. With the use of reliable decisions of sufficient span, we can effectively cancel this additional user completely, avoiding this loss of diversity.

We observed that under the circumstance where the two users are received with powers that are relatively close, it is best to use a full-coupled receiver structure in which the receiver for each user utilizes the decisions of the other user's receiver for interference mitigation, since both are experiencing relatively high interference. Under the circumstance where the received powers of the two users are disparate, it is best to use a half-coupling receiver structure in which the decisions of the stronger user are used in the mitigation of its interfering effects in the receiver processing of the weaker user. This strategy is used to form an adaptively structured receiver, which switches from fullcoupling to half-coupling, depending upon the received power conditions of the users.

This adaptively structured receiver is simulated for a two element antenna array and frequency selective channel conditions with a pedestrian fading rate of $4 \mathrm{~Hz}$. Under these conditions, acceptable performance was maintained down to $E_{s} / N_{o}=16 \mathrm{~dB}$ and a $C / C=-24 \mathrm{~dB}$. A four element diversity array receiver was simulated under fre- quency selective channel conditions with fading at a rate of $81 \mathrm{~Hz}$. This receiver was found to maintain acceptable performance for $E_{s} / N_{o}$ down to $22 \mathrm{~dB}$ and a $C / C$ down to $-12 \mathrm{~dB}$, proving to be a viable SDMA receiver for the given TDMA slot transmission format.

\section{References}

[1] H.P. Lin, S.S. Jeng, I. Parra, G. Xu, W.J. Vogel, and G.W. Torrence, "Experimental studies of SDMA schemes for wireless communications", in Proceedings of ICASSP, pp. 1760-1763, 1995.

[2] J. Fuhl and A.F. Molisch, "Capacity enhancement and BER in a combined SDMA/TDMA system", in Proceedings of the IEEE 46th Vehicular Technology Conference, pp. 1481-1485, 1996.

[3] A. Paulraj and C.B. Papadias, "Space-time processing for wireless communications", in Third Smart Antennas Workshop, 1996.

[4] J.H. Winters, J. Salz, and R.D. Gitlin, "The impact of antenna diversity on the capacity of wireless communication systems", IEEE Transactions on Communications, vol. 42, pp. 1740-1750, 1994.

[5] T.L. Fulghum, Adaptive Multielement Decision Feedback Receiver Structures for Narrowband Multiuser Detection in a Frequency Selective Channel, $\mathrm{PhD}$ thesis, North Carolina State University, May, 1998.

[6] G.E. Bottomley and K. Jamal, "Adaptive arrays and MLSE equalization", in Proceedings of the IEEE 45th Vehicular Technology Conference, pp. 50-54, 1995.

[7] A. Duel-Hallen, "A family of multiuser decisionfeedback detectors for asynchronous CDMA channels", IEEE Transactions on Communications, vol. 43, pp. 421-435, 1995.

[8] C. Tidestav, A. Ahlen, and M. Sternad, "Narrowband and broadband multiuser detection using a multivariable DFE", in Proceedings of PIMRC, pp. 732-736, 1995 .

[9] M. Uesugi, S. Futagi, and K. Homma, "Interference cancellation method using DFE", in Proceedings of the IEEE 46th Vehicular Technology Conference, pp. 1190-1194, 1996.

[10] Telecommunications Indus. Assoc., EIA/TIA Interim Standard: Cellular System Dual-Mode Station-Base Station Compatibility Standard, 1992.

[11] P. Dent, G.E. Bottomley, and T. Croft, "Jakes model revisited", Electronics Letters, vol. 29, pp. 1162-1163, 1993. 